\title{
Multiple vaginal examinations and early neonatal sepsis
}

\author{
Usha Christopher ${ }^{1}$, Goldy S. J. ${ }^{1 *}$, Bewin Oral J. ${ }^{2}$, Adlin Rose C. ${ }^{1}$
}

\begin{abstract}
${ }^{1}$ Department of Obstetrics and Gynecology, ${ }^{2}$ Department of Microbiology, Dr. Somervell Memorial CSI Medical
\end{abstract} College and Hospital, Karakonam, Kerala, India

Received: 09 February 2019

Accepted: 15 February 2019

\section{*Correspondence:}

Dr. Goldy S. J.,

E-mail: paulwilson555@gmail.com

Copyright: (C) the author(s), publisher and licensee Medip Academy. This is an open-access article distributed under the terms of the Creative Commons Attribution Non-Commercial License, which permits unrestricted non-commercial use, distribution, and reproduction in any medium, provided the original work is properly cited.

\section{ABSTRACT}

Background: Early onset neonatal sepsis (EONS) is caused mainly by organisms present in the genital tract. Maternal risk factors increase the incidence of EONS. This study was done to find out the association between one such risk factor i.e., multiple vaginal examinations and EONS.

Methods: Case control study. 114 patients with three or more vaginal examinations after rupture of membranes were taken as cases and 114 patients with less than three vaginal examinations after rupture of membranes were taken as controls. All these babies were followed up for the development of EONS.

Results: Of the 114 cases, 6 babies developed EONS. None of the babies in the control group developed EONS. So, 3 or more vaginal examinations after rupture of membranes in labour is significantly associated with early onset neonatal sepsis with p-value of 0.01305 .

Conclusions: Multiple vaginal examinations after rupture of membranes is a risk factor for early onset neonatal sepsis.

Keywords: EONS, Rupture of membranes, Vaginal examination

\section{INTRODUCTION}

Sepsis is one of the main causes of neonatal mortality. About $30-50 \%$ of the total neonatal deaths in developing countries is due to sepsis. ${ }^{1,2}$ It is estimated that about $20 \%$ of neonates develop sepsis and $1 \%$ die of sepsis related causes. ${ }^{2}$ So sepsis related mortality can be prevented with prevention of sepsis itself.

According to the data from National Neonatal Perinatal Database (NNPD, 2002-03) the incidence of neonatal sepsis is 30 per 1000 live births. The NNPD network in India found sepsis to be one of the commonest causes of neonatal mortality. Neonatal sepsis contributes to $19 \%$ of all neonatal deaths in India. ${ }^{3}$

Newborn sepsis is categorized as:

\section{Early onset neonatal sepsis (EONS)}

Presents within the first 72 hours of life and is acquired from the maternal genital tract during the course of labour.

\section{Late onset neonatal sepsis (LONS)}

Presents after 72 hours of life and is caused by organisms present in the hospital, community or home which is acquired after delivery.

Based on Indian studies, the following risk factors were associated with early onset neonatal sepsis: $:^{4,5}$

1. Low birth weight (<2500 grams)

2. Prematurity 
3. Febrile illness in the mother

4. Foul smelling liquor

5. Meconium stained liquor

6. Duration of rupture of membranes-more than 24 hours

7. Single unclean or more than 3 sterile vaginal examinations during labour

8. Prolonged labour-more than 24 hours

9. Perinatal asphyxia.

The amniotic cavity is nearly always sterile because fetal membranes form a protective sac around the fetus. When these membranes rupture, a route for infection to the fetus is formed. It is a common practice to do frequent vaginal examinations by the staff who takes care of women in labour. This increases the maternal and fetal infection. The causes for multiple vaginal examinations include failure to use the partograph and routine vaginal examinations at change of shift.

The 'gold standard' for assessing progress in labour is by performing vaginal examinations (VEs) and measuring cervical dilatation. ${ }^{6}$ Multiple VEs after prelabour rupture of membranes has been reported as an independent predictor of neonatal sepsis. ${ }^{7}$ Imseis et al. found a marked increase in the organisms isolated after VE in women with both ruptured and intact membranes. ${ }^{8}$

Warren found that VEs have become routine in labour that they are no longer seen as an intervention. ${ }^{9}$ It is difficult to know whether routine VEs are really needed, with the lack of evidences. In spite of these concerns, there is an over use of VEs in labour. ${ }^{10}$

The World Health Organisation (WHO) insists that the VEs should be done only when absolutely necessary and preferably one vaginal examination to diagnose active labour. ${ }^{11}$ Stuart pointed out that very less research evidences are available to determine the number of VEs that should be done in labour. ${ }^{12}$ The timing and frequency of VEs has not changed much for a long time. Even now most of the hospitals recommend vaginal examinations every four hours. ${ }^{13}$

Borders et al found that a woman in labour had an average of 4 vaginal examinations. ${ }^{14}$ This is definitely more than the recommendations by the World Health Organization, which recommends vaginal examination once in every 4 hours during the first stage. There is no evidence to agree the increased frequency of VEs which is being practised at present. But current evidence states that multiple VEs in labour increase the risk for chorioamnionitis and in turn neonatal sepsis. Border found that in a group of low-risk healthy women, the rate of chorioamnionitis was $6 \%$. The average VEs in these women was seven.

When the duration of labour increased, the number of VEs done also increased proportionately. When 4 hourly VEs criteria was applied, about $70 \%$ had more VEs than recommended. ${ }^{15} \mathrm{~A}$ study found that the incidence of chorioamnionitis has increased from $2.7 \%$ to $6.0 \%$ when singleton births from 1995-2010 were analysed. ${ }^{16}$

VE frequency is the only risk factor which can be controlled by healthcare providers. This present study was conducted in a tertiary care centre to find out the association between multiple vaginal examinations and EONS.

The aim of this study is to investigate the association between multiple VEs and early onset neonatal sepsis.

\section{METHODS}

It was a case control study.

\section{Inclusion criteria}

- $\quad$ Patients who had three or more vaginal examinations after rupture of membranes were taken as cases.

- The next patient without any risk factors for sepsis was taken as control.

\section{Exclusion criteria}

- Gestational age less than 28 weeks, baby weight less than 1000 grams, lethal congenital anomalies in baby.

\section{These alleged risk factors were:}

1. Three or more vaginal examinations after rupture of membranes

2. Intrapartum fever

3. Foul smelling liquor

4. Untreated maternal urinary tract infection.

Cord blood was sent for total and differential white cell counts for all cases. Assessment of the newborn was done at birth and followed up by the neonatologist. Blood culture was sent at 2-4 hours of age for all these babies according to the Neonatology Department protocol. After 24 hours, total and differential white cell counts were repeated in the peripheral blood. Blood culture and counts were sent for babies born to controls only if clinically indicated. For 48 hours all babies were observed for signs of sepsis. Maternal and neonatal data were collected from case records. Neubauer counting chamber was used for total white cell counts. On peripheral blood smears stained with Leishman stain, differential counts were done. From white cell counts, absolute neutrophil counts and immature: total neutrophil ratios were calculated.

For blood culture, $2 \mathrm{ml}$ of blood was inoculated into two blood culture bottles. One culture bottle was containing Biphasic Mackonkey medium (BPMM) and the other containing Brain Heart Infusion (BHI) broth. After inoculation, the bottles were sent to the microbiology 
laboratory where they were incubated at $37^{\circ} \mathrm{C}$ for 48 hours. These bottles were checked for growth up to 14 days if there was no growth by 48 hours.

\section{Criteria for neonatal sepsis}

Proven neonatal sepsis was diagnosed when blood culture was positive. In the absence of a positive blood culture, clinical signs and investigations were considered. Probable neonatal sepsis was diagnosed, when there were one or more signs of sepsis along with two or more positive laboratory parameters.

The signs of neonatal sepsis include:

1. Lethargy

2. Poor feeding

3. Temperature changes $\left(<36^{\circ} \mathrm{C}\right.$ or $\left.>37.8^{\circ} \mathrm{C}\right)$ for more than one hour (checked in axilla)

4. Jaundice (severe unconjugated/conjugated)

5. Apnoea

6. Any respiratory distress (>6-12 hours age)

7. Poor peripheral perfusion (capillary filling time $>5$ sec)

8. Tachycardia $>160 /$ min

9. Gastrointestinal manifestations (vomiting, diarrhoea, ileus)

10. Skin manifestations (petechiae, paronychia, omphalitis)

11. Central nervous system manifestations (high pitched cry, bulging fontanelle, seizures)

12. Bleeding diathesis.

The laboratory parameters were:
1. Total white cell count $<5000 / \mathrm{mm}^{3}$ or $>20,000 / \mathrm{mm}^{3}$

2. Absolute neutrophil count $<1500 / \mathrm{mm}^{3}$

3. Immature: total neutrophil ratio $>0.2$

4. Platelets $<150,000 / \mathrm{mm}^{3}$

5. Evidence of infection on blood picture (toxic granules, cytoplasmic vacuolation).

Newborns that fulfilled the criteria for proven or probable sepsis were considered positive for sepsis. Other babies were considered negative. According to the guidelines of the Neonatology Department, antibiotics were given.

\section{Statistical analysis}

Data were entered in Microsoft Excel and statistical analyses were done using SPSSPC+ software. Comparison of the different variables was done by Student's t-test, Chi-square test and Fisher's exact test. Data were analyzed to see the association between multiple VE and early onset neonatal sepsis.

\section{RESULTS}

One hundred and fourteen cases and controls were enrolled in this study. One hundred and fourteen cases had three or more than three vaginal examinations after rupture of membranes in labour.

One hundred and fourteen controls had less than three vaginal examinations after rupture of membranes in labour. Cases and controls were analyzed as below. The comparability of samples of case and control in relation to age was tested. The test was done using student's ttest.

Table 1: Age distribution.

\begin{tabular}{|l|l|l|l|l|l|}
\hline Variables & Group & N & Mean (years) & Std. deviation & p-value \\
\hline \multirow{2}{*}{ Age } & Case & 114 & 24.36 & 3.83 & 0.2279 \\
\cline { 2 - 6 } & Control & 114 & 25.02 & 4.71 & \\
\hline
\end{tabular}

Table 2: Gestational age distribution.

\begin{tabular}{|l|l|l|l|l|l|}
\hline Variables & Group & N & Mean (weeks) & Std. deviation & p-value \\
\hline \multirow{2}{*}{ Gestational age } & Case & 114 & 39.25 & 1.47 & 0.4458 \\
\cline { 2 - 6 } & Control & 114 & 39.11 & 1.12 & \multirow{2}{*}{} \\
\hline
\end{tabular}

Table 3: Distribution of parity.

\begin{tabular}{|l|l|l|l|l|}
\hline Parity & Case $(\mathbf{N})$ & Control $(\mathbf{N})$ & p-value & $\chi^{2}$ \\
\hline Nulliparous & 95 & 67 & 0.0000 & 16.718 \\
\hline Parous & 19 & 47 & & \\
\hline
\end{tabular}

The $95 \%$ confidence interval of the differences is from 1.7330 to 0.4172 . The p-value is not significant. There is no significant difference in the distribution of age between case and control (Table 1). The comparability of samples of case and control in relation to gestational age was tested using student's t-test (Table 2). 
The $95 \%$ confidence interval of the differences is from 0.2092 to 0.4723 . The p-value is not significant. There is no significant difference in the distribution of gestational age between case and control. The comparability of samples of case and control in relation to parity was tested using Chi-square test. The distribution of parity among cases and controls is given in Table 3 . The pvalue is significant. More of nulliparous women had 3 or more than 3 PVs in labour. The comparability of samples of case and control in relation to birth weight of babies was tested using student's t-test (Table 4).

The $95 \%$ confidence interval is from 8.7332 to 231.6002 . The p-value is significant. Birth weights of babies were significantly more in cases when compared to controls. The comparability of samples of case and control in relation to duration of rupture of membranes (ROM) was tested using student's t-test (Table 5).

Table 4: Birth weights of babies.

\begin{tabular}{|l|l|l|l|l|l|}
\hline Variables & Group & N & Mean (grams) & Std. deviation & p-value \\
\hline \multirow{2}{*}{ Birth weight } & Case & 114 & 3104.73 & 439.06 & 0.0347 \\
\cline { 2 - 6 } & Control & 114 & 2984.56 & 414.48 & 0.967 \\
\hline
\end{tabular}

Table 5: Duration of ROM.

\begin{tabular}{|c|c|c|c|c|c|}
\hline Variables & Group & $\mathbf{N}$ & Mean (hours) & Std. deviation & p-value \\
\hline \multirow{2}{*}{ Duration of ROM } & Case & 114 & 19.83 & 12.36 & \multirow{2}{*}{0.000000} \\
\hline & Control & 114 & 5.36 & 4.69 & \\
\hline
\end{tabular}

Table 6: Duration of labour.

\begin{tabular}{|l|l|l|l|l|l|}
\hline Variables & Group & N & Mean (hours) & Std. deviation & p-value \\
\hline \multirow{2}{*}{ Duration of labour } & Case & 114 & 14.15 & 7.00 & 0.000003 \\
\cline { 2 - 6 } & Control & 114 & 9.90 & 6.12 & 0.12 \\
\hline
\end{tabular}

The $95 \%$ confidence interval of the differences is from 12.0676 to 16.8623 . The p-value is significant. The mean duration of ROM is significantly more in case than of control.

The comparability of samples of case and control in relation to duration of labour was tested using student's ttest (Table 6). The $95 \%$ confidence interval of the differences is from 2.5417 to 5.9496 . The $p$-value is significant. The mean duration of labour is significantly more in case than of control.

Table 7: Number of PVs among cases.

\begin{tabular}{|l|l|}
\hline Number of PVs & Number of cases \\
\hline 3 & 51 \\
\hline 4 & 32 \\
\hline 5 & 16 \\
\hline 6 & 6 \\
\hline 7 & 2 \\
\hline 8 & 2 \\
\hline 9 & 1 \\
\hline 10 & 1 \\
\hline 11 & 1 \\
\hline 13 & 1 \\
\hline 16 & 1 \\
\hline Total & 114 \\
\hline
\end{tabular}

Table 8: Number of PVs among controls.

\begin{tabular}{|l|l|}
\hline Number of PVs & Number of controls \\
\hline 0 & 37 \\
\hline 1 & 55 \\
\hline 2 & 22 \\
\hline Total & 114 \\
\hline
\end{tabular}

Among the 114 cases, only two babies had positive blood culture. One had grown Group B streptococci while other had grown coagulase negative staphylococci. The number of vaginal examinations after rupture of membranes in the first case is three and the number of vaginal examinations after rupture of membranes in the second case is four.

Table 9: Proven sepsis.

\begin{tabular}{|l|l|}
\hline Culture positive & Number of PVs after ROM \\
\hline $1^{\text {st }}$ baby & 3 \\
\hline $2^{\text {nd }}$ baby & 4 \\
\hline
\end{tabular}

Among the 114 cases, four babies had probable sepsis according to the criteria used. The number of vaginal examinations after rupture of membranes in these four cases were as given in Table 10. 
Table 10: Probable sepsis.

\begin{tabular}{|l|l|}
\hline Probable sepsis & Number of PVs after ROM \\
\hline $1^{\text {st }}$ baby & 3 \\
\hline $2^{\text {nd }}$ baby & 5 \\
\hline $3^{\text {rd }}$ baby & 3 \\
\hline $4^{\text {th }}$ baby & 3 \\
\hline
\end{tabular}

Of the 114 cases, 6 babies developed sepsis. Two were culture positive and four were culture negative but fulfilled the criteria for probable sepsis. All six cases were nulliparous. None of the babies in the control group developed sepsis. The results were analyzed with chi square test.

Table 11: Sepsis.

\begin{tabular}{|l|l|l|l|}
\hline Sepsis & Case & Control & p-value \\
\hline Yes & 6 & 0 & 0.01305 \\
\hline No & 108 & 114 & \\
\hline
\end{tabular}

The chi-square statistic is 6.1622 . The $\mathrm{p}$-value is 0.01305 . This result is significant at $\mathrm{p}<0.05$. So, 3 or more vaginal examinations after rupture of membranes in labour is significantly associated with early onset neonatal sepsis.

\section{DISCUSSION}

Early onset neonatal sepsis is caused mainly by organisms present in the genital tract. These infections are acquired by vertical transmission from the maternal genital tract during the intrapartum period. The fetus in utero is protected from infections by the maternal immune system and also by the placenta and membranes.

Several factors, both maternal and fetal can cause damage to these barriers, leading to neonatal sepsis. Maternal factors that predispose to neonatal infections are:

1. Prelabour rupture of membranes

2. Prolonged labour

3. Infected birth canal

4. Chorioamnionitis

5. Multiple vaginal examinations.

This study was performed to find the association between multiple vaginal examinations and early onset neonatal sepsis. Three and more than three vaginal examinations was taken as a risk factor based on the previous study results. ${ }^{4,5}$ One hundred and fourteen cases and controls were enrolled in this study. In this study, age and gestational age were comparable between cases and controls. Of the 114 cases, 95 (83.33\%) were nulliparous whereas in the control group only $67(58.77 \%)$ were nulliparous. This shows that the nulliparous women had more PVs in labour when compared to parous women.

In the present study, birth weights of babies were significantly more in cases when compared to controls.
When the birth weights increased, the duration of labour also increased which led to more vaginal examinations.

The mean duration of ROM was 19.82 hours in cases while the mean duration of ROM was 5.36 hours in controls. The mean duration of labour was 14.15 hours in cases and 9.90 hours in control. The duration of ROM and duration of labour were significantly more in cases than controls. As the duration of ROM and duration of labour increased, the number of PVs done also increased.

Among the 114 cases, only two babies had positive blood culture. Group B streptococci grew in one while Coagulase negative staphylococci grew in the other. The risk factors for sepsis in these babies were $3 \mathrm{PVs}$ after ROM in the first case and 4 PVs after ROM in the second case. Four babies had probable sepsis according to the criteria used. Of these four, three had 3 PVs after ROM and one had 5 PVs after ROM.

Of the 114 cases, 6 babies developed sepsis. Two were culture positive and four were culture negative but fulfilled the criteria for probable sepsis. All six cases were nulliparous. None of the babies in the control group developed sepsis. So, 3 or more vaginal examinations after rupture of membranes in labour is significantly associated with early onset neonatal sepsis with $\mathrm{p}$-value of 0.01305 .

As noted, multiple vaginal examinations in labour after ROM increase the risk of early onset neonatal sepsis. Vaginal examinations should be done only when it is absolutely necessary rather than doing it routinely every hour or two. Also, aseptic precautions should be taken for vaginal examinations. Vaginal examination should not be done on a woman with prelabour rupture of membranes if she is not in labour. Only a speculum examination should be done under strict asepsis. Thus, reducing the number of PVs after ROM will definitely bring down the incidence of EONS.

\section{CONCLUSION}

Early onset neonatal sepsis is a major cause of neonatal morbidity and mortality. Maternal risk factors play an important role in EONS. This study like other studies has shown a significant association between multiple vaginal examinations $(\geq 3)$ and early onset neonatal sepsis.

\section{ACKNOWLEDGMENTS}

Authors would like to thank faculty and staff of the Department of Obstetrics and Gynaecology and the Department of Microbiology, Dr. Somervell Memorial CSI Medical College and Hospital, Karakonam, Kerala.

\section{Funding: No funding sources}

Conflict of interest: None declared

Ethical approval: The study was approved by the Institutional Ethics Committee 


\section{REFERENCES}

1. Bang AT, Bang RA, Bactule SB, Reddy HM, Deshmukh MD. Effect of home-based neonatal care and management of sepsis on neonatal mortality: field trial in rural India. Lancet. 1999;354:1955-61.

2. Stoll BJ. The global impact of neonatal infection. Clin Perinatol. 1997;24:1-21.

3. Report of the National Neonatal Perinatal Database (National Neonatology Forum) 2002-03.

4. Singh $M$, Narang A, Bhakoo ON. Predictive perinatal score in the diagnosis of neonatal sepsis. J Trop Pediatr. 1994;40(6):365-8.

5. Takkar VP, Bhakoo ON, Narang A. Scoring system for the prediction of early neonatal infections. Indian Pediatr. 1974;11:597-600.

6. Phelps JY, Higby K, Smyth MH, Ward JA, Arredondo F, Mayer A. Accuracy and intraobserver variability of simulated cervical dilatation measurements. Am J Obstet Gynecol. 1995;173(3):942-5.

7. Seaward PG, Hannah ME, Myhr TL, Farine D, Ohlsson A, Wang EE, et al. International multicenter term PROM study: evaluation of predictors of neonatal infection in infants born to patients with premature rupture of membranes at term. Am J Obstet Gynecol. 1998;179(3):635-9.

8. Imseis HM, Trout WC, Gabbe SG. The microbiologic effect of digital cervical examination. Am J Obstet Gynecol. 1999;180:578-80.
9. Warren C. Why should I do vaginal examinations? Pract Midwifery. 1999;2(6):12-3.

10. Cheyne H, Hundley V, Dowding D, Bland JM, McNamee P, Greer I, et al. Effects of algorithm for diagnosis of active labour: cluster randomised trial. BMJ. 2008;337(7683):1396-400.

11. World Health Organisation. Safe motherhood - care in normal birth: a practical guide. Geneva: WHO; 1997.

12. Stuart M. Midwives' discourses on vaginal examination in labour. $\mathrm{PhD}$ thesis. University of West of England; 2008.

13. National Institute for Health and Clinical Excellence 2007 clinical guideline 55: intrapartum care.

14. Borders N, Lawton R, Martin SR. A clinical audit of the number of vaginal examinations in labor: a NOVEL idea. J Midwifery Womens Health. 2012;57(2):139-44.

15. Shepherd A, Cheyne H. The frequency and reasons for vaginal examinations in labour. Women Birth. 2013;26(1):49-54.

16. Getahun D. Temporal trends in chorioamnionitis by maternal race/ethnicity and gestational age (19952010). Int J Reprod Med. 2013;2013:1-6.

Cite this article as: Christopher U, Goldy SJ, Oral BJ, Rose AC. Multiple vaginal examinations and early neonatal sepsis. Int J Reprod Contracept Obstet Gynecol 2019;8:876-81. 